\begin{tabular}{ll} 
O P E R A T I O N S R E S E A R C H A N D D E C I S I O N S \\
\hline No. 2
\end{tabular}

DOI: $10.37190 /$ ord200201

\title{
THE OPTIMISATION OF INSURANCE CONTRACTS ON THE VIATICAL MARKET
}

\author{
JOANNA DĘBICKA*, STANISŁAW HEILPERN \\ Wrocław University of Economics and Business, \\ Komandorska 118/120, 53-345 Wrocław, Poland
}

\begin{abstract}
We focus on ensuring the financial requirements of a person that has life insurance and needs money because of suffering from a terminal illness that requires costly diagnosis and treatment. On the secondary market of life insurance (the viatical market), companies offer purchase of rights to benefits after the death of the insured. The paper aims to analyse the problem of optimising the life settlement for the insured. We determine the amount of the nominal value of the benefit which the insured intends to sell in such a way so that the number of benefits and premiums maximise the average amount of funds available to the insured. We use various approaches of the insured to risk to find an optimal solution, which also allows taking into consideration the different kinds of human behaviour in risky conditions. The obtained theoretical results are illustrated with examples demonstrating the possibility of their application in practice.
\end{abstract}

Keywords: viatical settlement, multistate model, Bernoulli principle, rank dependent expected utility theory, cumulative prospect theory.

\section{Introduction}

Nowadays, behavioural finance has begun to attract increasing attention in the insurance theory. The generalisation of utility theory (like ranked dependent expected utility theory using the risk measures based on distorted probabilities or the perspective theory) has become the basis to define extended behavioural premium principles $[9,13]$. In particular, a premium principle under the cumulative prospect theory which extends the equivalent utility principle, along with a literature review on this subject, can be found in [14]. Authors of publications in this field pay attention to the interesting possibility of studying the decision problem also from the viewpoint of the insured willing

\footnotetext{
* Corresponding author, email address: joanna.debicka@ue.wroc.pl Received 10 December 2019, accepted 23 September 2020
} 
to buy insurance contract. In this paper, we focus on contracts concluded on the secondary insurance market and decisions on the resale of rights to the insurance benefit for the policyholder. No literature deals with the problem of making optimal decisions with concluding contracts in the viatical insurance market (a part of the secondary insurance market). The present paper fills this gap.

A significant problem is ensuring the financial requirements of a person (often a widow or a widower) having life insurance. Such activities are typically associated with a terminal illness that requires a costly diagnosis and treatment. The easiest form of receiving money from life insurance is to withdraw from the contract of insurance. Then the insurer is obliged to pay the surrender value. It appears that there are other possibilities for the insured to receive prior financial gratification from life insurance. One of them is to buy an insurance contract with the additional option that allows the insured to obtain the death benefit when he is still alive (accelerated death benefits - ADBs), for example, when the insured is terminally ill. The beneficiaries may still receive a benefit after the death of the insured, although it is reduced by the amount which was previously paid. Sometimes insurance companies allow the insured to rebuy the option of accelerated payment of death benefits after the diagnosis of the disease. This flexibility is stimulated by the strongly growing secondary market for life insurance (the viatical market). The insured can sell his/her policy for an amount that is greater than the surrender value (and less than the death benefit). Then the viatical company takes overpaying the insurance premiums, and in case of death of the insured gets the death benefits. Such agreements are offered to people who have developed a terminal disease (viatical settlement); see, e.g., $[1,7,15]$.

Sloan and Norton [19] show that the decision to buy long-term care insurance is affected by personal risk aversion and expectations. The choice of resale rights to the death benefit also depends on the insured risk aversion. But the situation of the insured person is different from that in case of buying or not buying long-term care insurance. The viatical contract does not meet any specific needs and does not protect the policyholder against such financial loss as reimbursement of medical or other expenses incurred. The insured can use the obtained benefits for any purpose.

The realisation of the viatical contract is connected with cash flows. In this paper, we analyse the cash flows arising from such agreements from the points of view of the insured. The owner of the life insurance policy wants to maximise his benefits. If an insured person resells his right to the policy, (s)he obtains the viatical settlement payment $(V S P)$ from the investor. Under conditions of the whole-life insurance contract in the event of death, the insurer pays the death benefit $c$ to the policyholder (an endowed person). The policyholder is obliged to pay annual premiums $p$ at the beginning of each year of the insurance period. We assume that the insured becomes terminally ill, and he wants to resell the rights to the death benefit. The insured consider the possibility to resell part $\alpha$ of the rights to death benefit $c$. By $\alpha \in[0,1]$ we denote the so-called resale 
parameter. $\alpha=0$ means that the viatical contract has not been completed. If $0<\alpha<1$, then these insured resell a part of rights to the death benefit. $\alpha=1$ implies that the investor bought the rights to the whole death benefit. The structure of cash flows generated after signing the viatical contract is shown in Fig. 1.

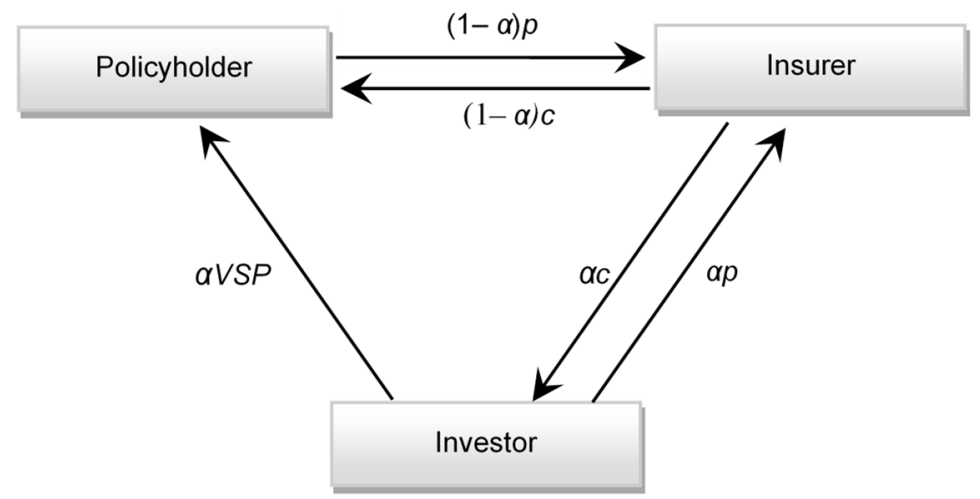

Fig. 1. A scheme of cash flows arising from both a viatical and insurance contract

This article aims to choose $\alpha$ for maximising the insured's (policyholder's) profit. We start the problem of optimising the viatical contract for the insured with determining the amount of the nominal value of the benefit which the insured intends to sell in such a way so that the number of benefits (derived from a viatical settlement payment and the insurance contract) and premiums maximise the average amount of funds available to the insured. Then, we suggest the Bernoulli principle to find an optimal solution (which maximises the expected discounted utility of cash flows in such a transaction), which also allows taking into consideration the different kinds of human behaviour under risky conditions. In the next step, we generalise this rule by taking into account the insured's subjective attitudes to the values of probability and the fact that they treat profits and losses in different ways. We apply the results of the rank dependent expected utility theory (RDEUT) [17, 9], and the cumulative prospect theory (CPT) [20, 13].

The paper is organised as follows. In Section 2, we describe the multiple state model and its probabilistic structure for viatical contracts. We derive formulas for the expected utility values of the total cash flows arising from such a contract (the insured expected profit) that takes into account the subjective attitude of the insured to the risk and the fact that people treat profits and losses differently. In Section 3, we maximise the expected utility values of the cash flow for the insured concerning the value of the resale parameter and the range of the viatical settlement payment. Theoretical results are illustrated by the empirical analysis of viatical contracts for the insured suffering from lung cancer. Numerical results are based on the actual data for the Lower Silesian Voivodship in Poland. Conclusions are included in Section 4. 


\section{Insured's expected profit}

Multiple state modelling is a classical stochastic tool for designing and implementing insurance products [8]. To model viatical contracts, we use multiple state model introduced in [4], which is presented in Fig. 2 (circles represent the states and arcs correspond to direct transitions between them). The set space $S$ of the multistate model consists of the following states:

1) the insured is terminally sick for a year after diagnosis,

2) the insured is terminally sick for the second year after diagnosis,

3 ) the insured is terminally sick for the third year after diagnosis,

$n$ ) the insured is terminally sick for the fourth year after diagnosis, $n+1)$ the insured dies, being terminally sick with a dread disease.

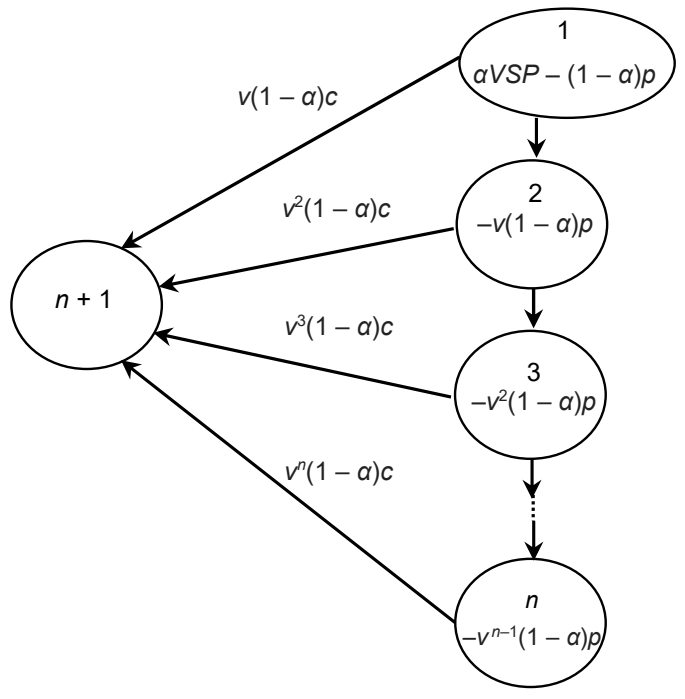

Fig. 2. A multiple state model for a viatical contract (based on [4])

In Figure 2, we mark the actual value (at the moment of concluding the viatical contract) of cash flows related to the transition between states, where $c$ is a given lump sum (death benefit), $\alpha$ is a resale parameter, and $v$ is a discount factor. The investor's commitments to the insured (VSP) and the commitments of the insured to the insurer (the actual value of the remaining part of the period premium $p$ ) are marked in circles.

Note that in states $\{1,2, \ldots, n\}$ remission of the disease is very unlikely. Moreover, note that the states are reflex and strictly transitional, and after one unit of time the insured risk leaves this state. Such a division of the state space and state properties is necessary because the probability of death for a dread disease by the sufferer depends on the duration of the disease, diagnosis, and the disease stage. 
We assume that an $x$-year-old person bought the whole-life insurance. This type of contract lasts $\omega-x$ years, where $\omega$ is the maximum age (according to life tables 100 or 110 years). Through the insurance period, the insured becomes terminally ill, and (s)he wants to resell the rights to the death benefit. Let $t(0<t \leq \omega-x)$ be a year of the insurance period in which the insured became seriously ill (it means that an $x+t$-year-old insured person became ill). Note that, because states from 1 to $n$ are reflex states, the term of the viatical contract is equal to $n$ years. Let the evolution of the insured risk be described by a discrete-time stochastic process $\left\{Y_{t}(k) ; k=0,1,2,3, \ldots, n\right\}$. We assume that process $\left\{Y_{t}(k)\right\}$ is a nonhomogeneous Markov chain and the transition probability from state $i$ to state $j((i, j \in S))$ in the $k$-th year of the contract is defined as follows:

$$
p_{i j}(t ; k)=P\left(Y_{t}(k+1)=j \mid Y_{t}(k)=i\right) P
$$

The transition probabilities can be determined using a multiple increment-decrement table (or multiple state life table) for particular dread diseases.

The insured considers the possibility to resell only part $\alpha$ of the rights to the death benefit. He would like to choose the parameter $\alpha$ for maximum benefit from his point of view. Note that, depending on the time of death of the insured person, there are four possible ways of realisation of a viatical contract (see Fig. 2). The probabilities of realisation of each are presented in Table 1 , where $p_{12}=p_{12}(t, 0), p_{23}=p_{23}(t, 1)$, $p_{34}=p_{34}(t, 2), \ldots, p_{n-1 n}=p_{n-1 n}(t, n-2)$.

Table 1. Possible realisations of a viatical contract

\begin{tabular}{|l|c|c|c|c|c|}
\hline Realisation & $1 \rightarrow n+1$ & $1 \rightarrow 2 \rightarrow n+1$ & $1 \rightarrow 2 \rightarrow 3 \rightarrow n+1$ & $\ldots$ & $1 \rightarrow 2 \rightarrow 3 \rightarrow \ldots \rightarrow n+1$ \\
\hline Probability & $q_{1}=1-p_{12}$ & $q_{2}=p_{12}\left(1-p_{23}\right)$ & $q_{3}=p_{12} p_{23}\left(1-p_{34}\right)$ & $\ldots$ & $q_{n}=p_{12} p_{23} \times \ldots \times p_{n-1 n}$ \\
\hline
\end{tabular}

Let $X$ be the random variable describing the total cash flows after a resale policy. The initial cash flow $a_{0}$ equals

$$
a_{0}=\alpha V S P-(1-\alpha) p
$$

because at the moment of conclusion of the viatical contract $(k=0)$, the insured obtains from the investor a part of viatical settlement payment $\alpha V S P$, but (s)he is obliged to pay a part of the premium for this part of the death benefit, which (s)he did not resell to the investor. Thus, the random variable $X$ can take the following values

$$
x_{1}=a_{0}+v(1-\alpha) c
$$




$$
x_{k}=a_{0}+v^{k}(1-\alpha) c-(1-\alpha) p \sum_{i=1}^{k-1} v^{i} \text { for } k=2,3, \ldots, n
$$

The distribution of the random variable $X$ takes the following form:

$$
P\left(X=x_{k}\right)=q_{k}
$$

Note that the values $x_{k}$ of the cash flow take the form

$$
x_{k}=\alpha V S P+(1-\alpha) b_{k}
$$

where $b_{k}=v^{k} c-p \sum_{i=0}^{k-1} v^{i}$. We have

$$
b_{k-1}-b_{k}=v^{k-1}(c(1-v)+p)>0
$$

for $k=2,3, \ldots, n$, then $b_{1}>b_{2}>\ldots>b_{n}$ and $x_{k}$ satisfy similar inequities. If

$$
b_{n}=v^{n} c-\left(1+v+v^{2}+\ldots+v^{n-1}\right) p>0
$$

then $x_{k}>0$ for every $k=1,2, \ldots, n$. So, the expected value of this cash flow is equal

$$
E X=\sum_{k=1}^{n} x_{k} q_{k}=\sum_{k=1}^{n}\left(\alpha V S P+(1-\alpha) b_{k}\right) q_{k}=\alpha V S P+(1-\alpha) \sum_{k=1}^{n} b_{k} q_{k}
$$

There is a vast literature on different kinds of human behaviours under risky conditions, and severe illness is such a situation. Thus the insured's optimal decisions should be analysed under this consideration. We consider the Bernoulli principle to find an optimal solution (which maximises the expected discounted utility of cash flows in such a transaction). Moreover, we generalise this rule by taking into account the insured's subjective attitudes to the values of probability and the fact that people treat profits and losses in different ways. Therefore, we also use the results of RDEUT and CPT.

The rank dependent expected utility theory (RDEUT) is based on the Choquet integral concerning the distorted probabilities [17]. The insured acts are guided by his/her utility function $u(x)$ and distorted function $w(q)$ in this case. The function $w:[0,1] \rightarrow[0,1]$ is increasing and it satisfies conditions: $w(0)=0$ and $w(1)=1$. The value of the expected utility of cash flow RDEUT is equal to 


$$
E_{w}(u(X))=\int_{0}^{\infty} w(S(x)) d x-\int_{-\infty}^{0}(1-w(S(x))) d x
$$

where $S(x)=1-F(x)$ is a survival function of random variable $u(X)$, and $F(x)$ is a cumulative distribution function of $u(X)$.

In our case, we have $b_{1}>b_{2}>\ldots>b_{n}$. This also implies that $x_{1}>x_{2}>\ldots>x_{n}$. Thus, we obtain

$$
\begin{aligned}
E_{w}(U(X))= & U\left(x_{1}\right) w\left(q_{1}\right)+U\left(x_{2}\right)\left(w\left(q_{1}+q_{2}\right)-w\left(q_{1}\right)\right)+U\left(x_{3}\right)\left(w\left(1-q_{4}\right)\right. \\
& \left.\left.-w\left(q_{1}+q_{2}\right)\right)+\ldots+U\left(x_{n}\right) 1-w\left(1-q_{n}\right)\right)=\sum_{k=1}^{n} U\left(x_{k}\right) w_{k}
\end{aligned}
$$

where $w_{1}=w\left(q_{1}\right), w_{2}=w\left(q_{1}+q_{2}\right)-w\left(q_{1}\right), w_{3}=w\left(1-q_{4}\right)-w\left(q_{1}+q_{2}\right) \ldots$ and $w_{n}$ $=1-w\left(1-q_{n}\right)$.

We have $\sum_{k=1}^{n} w_{k}=1$ in this case.

When the insured follows the RDEUT [16], he will maximise the expected utility of cash flow

$$
E_{w}(U(X))=\sum_{k=1}^{n} U\left(x_{k}\right) w_{k}
$$

We have

$$
x_{k}=a_{0}+\sum_{i=1}^{k-1}(\alpha-1) p v^{i}+(1-\alpha) c v^{k}
$$

so we use the discounted utility model $[10,18]$ in this case. The global utility $U$ is the sum of utilities $u$ of cash flow in each period discounted at rate $r$ in this model. So, we obtain

$$
U\left(x_{k}\right)=u\left(a_{0}\right)+u((\alpha-1) p) \sum_{i=1}^{k-1} v^{i}+u((1-\alpha) c) v^{k}
$$

We assume that the insured has the risk aversion, then his/her utility function $u$ is a continuous, concave, increasing function, such that $u(0)=0$. The expected utility of cash flow takes the following form: 


$$
E_{w}(U(X))=u_{0}+u_{p} A_{p}+u_{c} A_{c}
$$

where $u_{0}=u\left(a_{0}\right), u_{p}=u((\alpha-1) p), u_{c}=u((1-\alpha) c)$

$$
\begin{gathered}
A_{p}=\sum_{k=2}^{n}\left(\sum_{i=1}^{k-1} v^{i}\right) w_{k} \\
A_{c}=\sum_{k=1}^{n} v^{k} w_{k}
\end{gathered}
$$

The expected utility $E_{w}(U(X))$ is a concave function, too. When $w(q)=q$, we obtain the general utility theory [16] and $w_{k}=q_{k}$ in this case.

CPT, created by Tversky and Kahneman in [20], is another generalised utility theory. The insured, who follows this theory, treats positive and negative value differently. $\mathrm{He} / \mathrm{she}$ acts guided by his utility function $u(x)$ and two distorted functions $w_{+}(q), w_{-}(q)$. $\mathrm{He} / \mathrm{she}$ selects parameter $\alpha$, which maximises the generalised expected utility

$$
E_{w_{+}, w_{-}}(u(X))=\int_{0}^{\infty} w_{+}(S(x)) d x-\int_{-\infty}^{0} w_{-}(F(x)) d x
$$

This is a so-called symmetric integral [3].

We can see that it is a similar situation to the one in RDEUT and the general utility theory for maximization.

In practice, contracts are concluded for a maximum of 4 years on the secondary insurance market. Such a number of years results from the fact that an investor would typically like to rebuy the rights to the dead benefits from the insured whose maximum future lifetime is no longer than four years or, in some cases, two years (depending on medical circumstances). The difference results from the definition of a terminally ill person. On the one hand, the term "terminally sick" in the context of health care refers to a person who is suffering from a severe illness and whose life is not expected to go beyond two years at the maximum. On the other hand, for example, HIV+ patients with more than 4.5 years of life expectancy, are treated as patients in relatively good health. moreover, in numerical examples, multistate life tables are required to determine actuarial quantities. In this article, we use tables for people suffering from lung cancer, which falls into the group of tumours characterized by the highest morbidity and mortality rates. This deadly disease is a tumour with unfavourable prognosis because of relatively short survival time after the diagnosis. For this disease, the maximum survival time after the detection of so-called distant metastases is four years. Therefore, in the 
next sections of this article (without loss of generality), we will consider the 5-state model, i.e., the multiple state model for $n=4$, the scheme of which is shown in Fig. 2.

\section{Optimization of the insured's profit}

\subsection{Viatical settlement payment}

In this section, we will maximise the expected utility values of the cash flow for the insured, concerning the value of the parameter $\alpha$. The solution of such an optimisation problem depends on the value of the viatical settlement payment VSP. The VSP does not take any value. Its values are bounded from the bottom and the top. If the insured becomes terminally ill in the $t$ th year of the insured period, we obtain the following bounds for the values of VSP

$$
V(t)<V S P(t)<E B(t)+E C(t)
$$

where $V(t)$ is a net premium reserve of the insurance contract at moment $t, E C(t)$ is the investor's expected costs, and $E B(t)$ is its expected benefit at the beginning of the $t$-year of the insurance period [4].

Example 1. We assume that a 60 -year old insured man becomes terminally ill from lung cancer [6]. Thus, we take the transition probabilities $p_{i j}$ from actual multistate life tables for men [5]: $p_{12}=0.1717, p_{23}=0.4850$, and $p_{34}=0.0572$. Hence, the probabilities $q_{k}$ are equal to

$$
q_{1}=0.8283, \quad q_{2}=0.0884, \quad q_{3}=0.0785, \quad q_{4}=0.0048
$$

The range of $V S P$ depends on the age at entry. Let $c=100$ and premium interest rate be equal to $1 \%$. Then, if an insured bought the whole-life insurance when he was $x=20$ years old, then the period premium $p=1.4817$, but if he bought insurance when he was $x=55$ years old, then $p=4.2057$ (according to formula in [2] and Polish Life Table 2008 [11]). Moreover, let the investor's interest rate equal 2\% $(r=0.02$, then $v=0.980$ ). Namely, for an insurance contract bought by 20 years old man (after 40 years $t=40) 59.200<V S P<96.899$. If the insurance contract was signed, when the insured was 55 years old $(x=55)$, then (after 5 years $t=5) 14.254<V S P<93.482$.

\subsection{Rank dependent expected utility theory}

In this section, we assume that the insured follows RDEUT, and the utility function $u(x)$ has the power form. Theorem 1 to determine the optimum part of the resale rights to the death benefits from the insured's point of view. 
Theorem 1. We assume that insured has a whole life insurance contract with the annual premium $p$ and the death benefit $c$. Let the insured follows RDEUT with a utility function

$$
u(x)=(x+p) \beta-p \beta
$$

where $0<\beta \leq 1$ and with the distortion function

$$
w(q)=\frac{q^{\gamma}}{\left(q^{\gamma}+(1-q)^{\gamma}\right)^{1 / \gamma}}
$$

where $0<\gamma \leq 1$ describes the curvature of this function.

The most profitable decision for the insured depends on parameter $\beta$ :

A. If $0<\beta<1$, then the optimal resale parameter is equal to

$$
\alpha_{0}=\frac{\left(c A_{c}\right)^{1 /(\beta-1)}(c+p)}{\left((V S P+p)^{\beta}+p^{\beta} A_{p}\right)^{1 /(\beta-1)}+c^{\beta /(\beta-1)} A_{c}^{1 /(\beta-1)}}
$$

where $A_{p}$ and $A_{c}$ are given by (3) and (4), respectively, VSP is the viatical settlement payment.

B. If $\beta=1$, then we have

$$
\alpha_{0}=\left\{\begin{array}{llll}
0 & \text { for } & V S P> & \sum_{k=1}^{4} b_{k} w\left(q_{k}\right) \\
1 & \text { for } & V S P & <\sum_{k=1}^{4} b_{k} w\left(q_{k}\right)
\end{array}\right.
$$

where $q_{k}$ is the probability of terminating the viatical contract at $k$ th year, and

$$
b_{k}=v^{k} c-p \sum_{i=0}^{k-1} v^{i}
$$

If $V S P=\sum_{k=1}^{4} b_{k} w\left(q_{k}\right)$, then every $\alpha \in[0,1]$ is optimal.

\section{Proof}

Ad A. Note that applying (5) to (2), we obtain 


$$
E_{w}(U(X))=\alpha^{\beta}\left((V S P+p)^{\beta}+p^{\beta} A_{p}\right)+(c+p-\alpha c)^{\beta} A_{c}-p^{\beta}\left(1+A_{p}+A_{c}\right)
$$

and its derivative relative to $\alpha$ takes the form of

$$
E_{w}^{\prime}(U(X))=\beta \alpha^{\beta-1}\left((V S P+p)^{\beta}+p^{\beta} A_{p}\right)-c \beta(c+p-\alpha c)^{\beta-1} A_{c}
$$

This function for $0<\beta<1$ takes the value zero for parameter $\alpha$ equals

$$
\alpha_{0}=\frac{\left(c A_{c}\right)^{1 /(\beta-1)}(c+p)}{\left((V S P+p)^{\beta}+p^{\beta} A_{p}\right)^{1 /(\beta-1)}+c^{\beta /(\beta-1)} A_{c}^{1 /(\beta-1)}}
$$

The expected utility of cash flow is a concave function and $\alpha_{0}>0$, so we obtain the maximum at this point. This completes the proof (7).

Ad B. For $\beta=1$, we have $u(x)=x$ and we obtain (analogously to (1))

$$
E_{w}(U(X))=E_{w}(X)=\sum_{k=1}^{4} x_{k} w\left(q_{k}\right)=\alpha V S P+(1-\alpha) \sum_{k=1}^{4} b_{k} w\left(q_{k}\right)
$$

Note that (9) is a linear function of parameter $0 \leq \alpha \leq 1$. Then we obtain maximum $E_{w}(X)$ when $\alpha=0$ or $\alpha=1$. Thus, if $\operatorname{VSP}>\sum_{k=1}^{4} b_{k} w\left(q_{k}\right)$, we get maximum for $\alpha=1$. It means that the most profitable decision for the insured is to resell all rights to death benefit (the insured's expected profit is equal to viatical settlement payment VSP). If $V S P<\sum_{k=1}^{4} b_{k} w\left(q_{k}\right)$, we obtain maximum for $\alpha=0$. In such a situation, the most profitable decision for the insured is not to resell his insurance, because the maximum of the insured's expected profit equals $E X=\sum_{k=1}^{4} b_{k} w\left(q_{k}\right)$. When $V S P=\sum_{k=1}^{4} b_{k} w\left(q_{k}\right)$, all $\alpha$ 's are just as "good". This completes the proof (8).

Note that Theorem 1 consists of two extreme cases:

1. $\gamma=1$ and $0<\beta<1$ (Theorem 1A),

2. $\gamma=1$ and $\beta=1$ (Theorem 1B).

If $\gamma=1$, then the function $w$ does not distort the probabilities, and we obtain the classical utility theory (Corollary 1 ), the theory of maximisation of the expected value of cash flow (Corollary 2). 
Corollary 1 (utility theory). For $0<\beta<1$ (in (5)) and $\gamma=1$ (in (6)), we obtain the optimal resale parameter for the insured given by (7), where

$$
A_{p}=\sum_{k=2}^{4}\left(\sum_{i=1}^{k-1} v^{i}\right) q_{k} \text { and } A_{c}=\sum_{k=1}^{4} v^{k} q_{k}
$$

Corollary 2 (expected value of cash flow). For $\beta=1$ (in (5)) and $\gamma=1$ (in (6)), we obtain the optimal resale parameter for the insured, according to the following formula

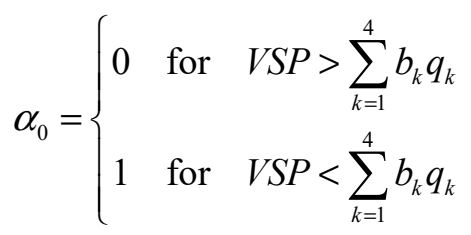

where $q_{k}$ is the probability of terminating the viatical contract at $k$ th year and

$$
b_{k}=v^{k} c-p \sum_{i=0}^{k-1} v^{i}
$$

If $V S P=\sum_{k=1}^{4} b_{k} w\left(q_{k}\right)$, then every $\alpha \in[0,1]$ is optimal.

We skip proofs of corollaries because they follow straightforwardly Theorem 1 and the considerations in Section 2.

Example 2 (RDEUT - example 1 continued). Let us assume that $\beta=0.6$ and $\gamma=0.61$, just as the authors suggest in [20]. The distorted probabilities $w_{k}$ are equal to

$$
w_{1}=0.6325, \quad w_{2}=0.1028, \quad w_{3}=0.2065, \quad w_{4}=0.0582
$$

and we obtain $A_{c}=0.9673, A_{p}=0.6695$. The expected utility of the cash flow for $x=20$ takes the form of

$$
E_{w}(U(X))=\left((V S P+1.4817)^{0.6}+0.8476\right) \alpha^{0.6}+0.9673(101.4817-100 \alpha)^{0.6}-3.3383
$$

and we obtain the maximum of RDEU of cash flow in

$$
\alpha_{0}=\frac{0.001071}{\left((V S P+1.4817)^{0.6}+0.8476\right)^{-2.5}+0.001087}
$$


Table 2 contains optimal values of $\alpha_{0}$ and RDEU of cash flow for different values of VSP. Figure 3 shows the graphs of these optimal values concerning the acceptable bounds of VSP.

Table 2. The values of optimal solution $\alpha_{0}$ and $E_{w}(U(X))$ for different $V S P$ values

\begin{tabular}{|c|c|c|c|c|c|}
\hline$V S P$ & $\alpha_{0}$ & $E_{w}(U(X))$ & $V S P$ & $\alpha_{0}$ & $E_{w}(U(X))$ \\
\hline 0 & 0.0071 & 12.1713 & 60 & 0.3895 & 15.4325 \\
\hline 10 & 0.0630 & 12.5290 & 70 & 0.4413 & 16.0939 \\
\hline 20 & 0.1311 & 13.0076 & 80 & 0.4878 & 16.7626 \\
\hline 30 & 0.2013 & 13.5582 & 90 & 0.5294 & 17.4347 \\
\hline 40 & 0.2690 & 14.1555 & 100 & 0.5666 & 18.1074 \\
\hline 50 & 0.3320 & 14.7839 & & & \\
\hline
\end{tabular}

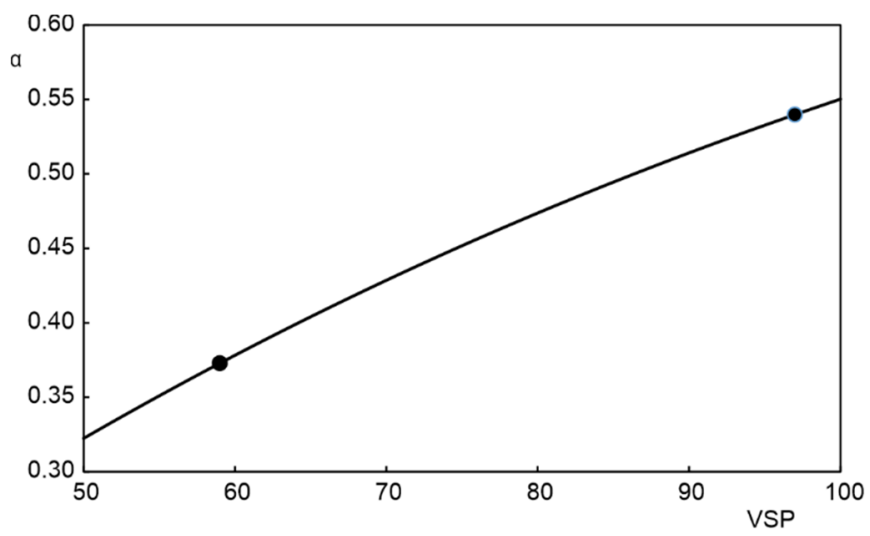

Fig. 3. The optimal values of the parameter $\alpha$ for different values of VSP

Higher values of the viatical settlement payment VSP imply the greater amount of the optimal part $\alpha$ of the rights to death benefit $c$. Figure 4 presents the values of $E_{w}(U(X))$ for different values of the parameter $\alpha$ and the fixed value $V S P=80$.

When $w(q)=q$, we obtain the classical utility theory (Corollary 1). In this case, $A_{c}=0.9754$ and $A_{p}=0.2529$. The expected utility of cash flow for $x=20$ takes the form

$$
E(U(X))=\left((V S P+1.4817)^{0.6}+0.3201\right) \alpha^{0.6}+0.9754(101.4817-100 \alpha)^{0.6}-2.8211
$$

and we obtain the maximum of the expected utility of cash flow in

$$
\alpha_{0}=\frac{0.00108}{\left((V S P+1.4817)^{0.6}+0.3201\right)^{-2.5}+0.001064}
$$




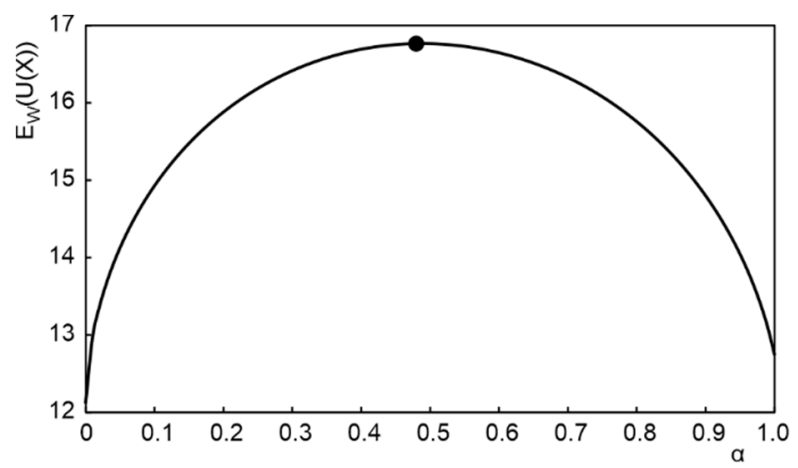

Fig. 4. The dependences of $E_{w}(U(X))$ on $\alpha$ for $V S P=80$

Table 3 contains the optimal values of $\alpha_{0}$ and the expected utility of cash flow for different values of $V S P$.

Table 3. The values of optimal solution $\alpha_{0}$ and $E(U(X))$ for different $V S P$ values

\begin{tabular}{|c|c|c|c|c|c|}
\hline$V S P$ & $\alpha_{0}$ & $E(U(X))$ & $V S P$ & $\alpha_{0}$ & $E(U(X))$ \\
\hline 0 & 0.0034 & 12.7965 & 60 & 0.3594 & 15.7562 \\
\hline 10 & 0.0479 & 13.0798 & 70 & 0.4120 & 16.3887 \\
\hline 20 & 0.1087 & 13.4983 & 80 & 0.4597 & 17.0323 \\
\hline 30 & 0.1744 & 13.9975 & 90 & 0.5027 & 17.6824 \\
\hline 40 & 0.2397 & 14.5506 & 100 & 0.5413 & 18.3358 \\
\hline 50 & 0.3018 & 15.1407 & & & \\
\hline
\end{tabular}

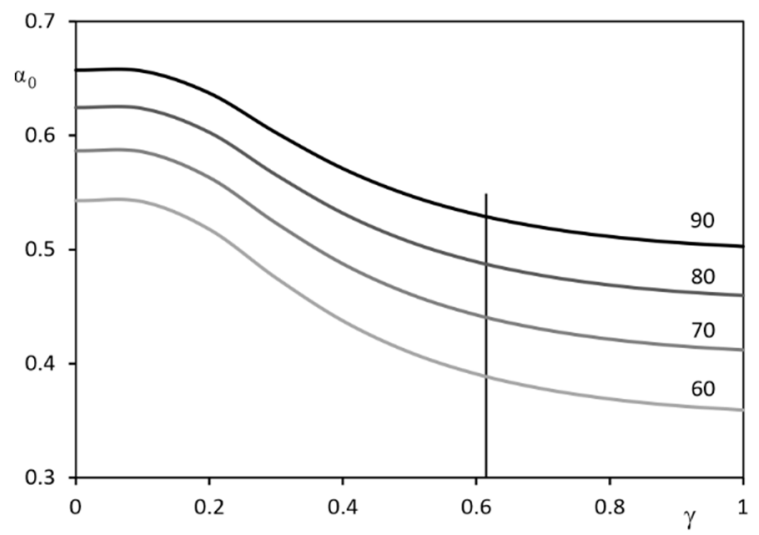

Fig. 5. The dependences of $\alpha_{0}$ on $\gamma$ for $V S P=60,70,80$, and 90

Now, we will study the impact of changing the value of parameter $\gamma$ on the optimal value of the resale parameter $\alpha_{0}$. In Figure 5, we present the plots of $\alpha_{0}$ vs. $\gamma$ for four values of $V S P$. 
Higher values of the parameter $\gamma$ imply the smaller value of the optimal $\alpha_{0}$, and the higher values of the viatical settlement payment VSP imply the greater value of $\alpha_{0}$. The shape of the curves is similar for various amounts of VSP. So, if the function $w$ distorts probabilities more ( $\gamma$ decreases), then the investor bought more rights to the death benefit ( $\alpha$ increases).

Until now, in the example, we assumed that $0<\beta<1$ (in particular $\beta=0.6$ ). If we assume that $\beta=\gamma=1$, then finding the optimal resale parameter consists of maximising the expected value of cash flow (Corollary 2). If $x=20$, then $59.200<V S P<96.899$, and the values of $b_{k}$ take the following form:

$$
b_{1}=96.558, \quad b_{2}=93.183, \quad b_{3}=89.874, \quad b_{4}=86.630 .
$$

Note that $b_{4}>0$, so all values $x_{k}>0$. Also, we have $\sum_{k=1}^{4} q_{k} b_{k}=95.687$ and the expected cash flow with respect to $V S P$ and $\alpha$ is equal to $E(X)=\alpha V S P+95.687(1-\alpha)$. Thus, for $59.200<V S P<95.687 \alpha=0$ guarantees the maximum value of the expected cash flow $E(X)$ and for $95.687<V S P<96.899$ we have $\alpha=1$. For $V S P=95.687$, every $0 \leq \alpha \leq 1$ is just as "good".

We can distinguish two extreme cases (looking at the parameter that distorts probability). First, if $\gamma=1$, then the function $w$ does not distort the probabilities and we obtain the classical utility theory. The value of optimal $\alpha 0$ is the smallest in this case. We obtain the highest amounts of $\alpha_{0}$ for a second extreme case when parameter $\gamma$ tends to 0 . In this limit case, we have the degenerate distorted function taking the form

$$
w(q)= \begin{cases}0 & \text { for } 0 \leq q<1 \\ 1 & \text { for } q=1\end{cases}
$$

The insured covers certain events only. We obtain $w_{1}=w_{2}=w_{3}=0, w_{4}=1$, $A_{p}=\sum_{k=1}^{3} v^{k}$ and $A_{c}=v^{4}$ in such a case. We can compute the limit values of $\alpha_{0}$ using equation (7).

\subsection{Prospect theory}

The utility function proposed by Kahneman and Tversky in [12] takes the form

$$
u(x)= \begin{cases}x^{\beta} & \text { for } x \geq 0 \\ -\lambda(-x)^{\beta} & \text { for } x<0\end{cases}
$$


where $0<\beta \leq 1$ and $\lambda \geq 1$. They accepted that $\beta=0.88$ and $\lambda=2.25$ based on an experiment. It is a concave function for the gains and convex for the losses. They also used the distorted function (2) with parameters $\gamma_{+}=0.61$ and $\gamma_{-}=0.69$.

If $b_{4}=v^{4} c-\left(1+v+v^{2}+v^{3}\right) p>0$, then $x_{k}>0$ for every $k=1,2,3,4$ (see Section 2), and we use the distorted probabilities $w_{+, k}$ only. In practice, this condition is usually satisfied because $c$ is much higher than $p$, so in this section we assume that $x_{4}>0$.

\section{Theorem 2}

A. If $0<\beta<1$, then the maximum value of the expected utility of cash flow can be assumed for the resale parameter

$$
\begin{gathered}
a=0, \alpha_{1}=\frac{B^{1 /(\beta-1)}-p(\lambda(V S P+p))^{1 /(\beta-1)}}{B^{1 /(\beta-1)}-\lambda^{1 /(\beta-1)}(V S P+p)^{\beta /(\beta-1)}}, \alpha_{V}=\frac{p}{V S P+p} \\
\alpha_{2}=\frac{p(V S P+p)^{1 /(\beta-1)}+B^{1 /(\beta-1)}}{(V S P+p)^{\beta /(\beta-1)}+B^{1 /(\beta-1)}} \text { or } 1
\end{gathered}
$$

where $B=A_{c} c^{\beta}-\lambda A_{p} p^{\beta}, A_{p}=\sum_{k=2}^{4}\left(\sum_{i=1}^{k-1} v^{i}\right) w_{+, k}$, and $A_{c}=\sum_{k=1}^{4} v^{k} w_{+, k}$.

B. If $\beta=1$, then the optimal resale parameter is equal to

$$
\alpha_{0}= \begin{cases}1 & \text { for } V S P+p>B \\ \alpha_{V} & \text { for } V S P+p<B \text { and } \lambda(V S P+p)>B \\ 0 & \text { for } \lambda(V S P+p)<B\end{cases}
$$

If $V S P+p=B$, then every $\alpha \in\left[\alpha_{V}, 1\right]$ is optimal and for $\lambda(V S P+p)=B$ every $\alpha \in$ $\left[0, \alpha_{V}\right]$.

\section{Proof}

Ad A. We have

$$
u\left(a_{0}\right)= \begin{cases}(\alpha V S P-(1-\alpha) p)^{\beta} & \text { for } \alpha \geq \alpha_{V} \\ -\lambda((1-\alpha) p-\alpha V S P)^{\beta} & \text { for } \alpha<\alpha_{V}\end{cases}
$$

$u((1-\alpha) c)=(1-\alpha) \beta c \beta$ and $u((\alpha-1) p)=-\lambda(1-\alpha) \beta p \beta$. So, if the insured follows the prospect theory, then the expected utility of the cash flow takes the following form:

$$
E(U(X))= \begin{cases}(\alpha V S P-(1-\alpha) p)^{\beta}+(1-\alpha)^{\beta} B & \text { for } \alpha \geq \alpha_{V} \\ (1-\alpha)^{\beta} B-\lambda((1-\alpha) p-\alpha V S P)^{\beta} & \text { for } \alpha<\alpha_{V}\end{cases}
$$


The first derivative with respect to $\alpha$ of the expected utility of the cash flow is equal to

$$
E^{\prime}(U(X))= \begin{cases}\beta(V S P+p)(\alpha V S P-(1-\alpha) p)^{\beta-1}-(1-\alpha)^{\beta-1} \beta B & \text { for } \alpha \geq \alpha_{V} \\ \lambda \beta(V S P+p)((1-\alpha) p-\alpha V S P)^{\beta-1}-(1-\alpha)^{\beta-1} \beta B & \text { for } \alpha<\alpha_{V}\end{cases}
$$

If $\alpha \geq \alpha_{V}$, then the first derivative of $E(U(X))$ is equal to zero for

$$
\alpha_{2}=\frac{p(V S P+p)^{1 /(\beta-1)}+B^{1 /(\beta-1)}}{(V S P+p)^{\beta /(\beta-1)}+B^{1 /(\beta-1)}}
$$

and if $\alpha<\alpha_{V}$, then we obtain $E^{\prime}(U(X))=0$ for

$$
\alpha_{1}=\frac{B^{1 /(\beta-1)}-p(\lambda(V S P+p))^{1 /(\beta-1)}}{B^{1 /(\beta-1)}-\lambda^{1 /(\beta-1)}(V S P+p)^{\beta /(\beta-1)}}
$$

Therefore, we ought to calculate the values of $E(U(X))$ at points $0, \alpha_{1}, \alpha_{V}, \alpha_{2}$, and 1 , and select the largest value. A maximum occurs for $\alpha$ chosen in this way.

Ad B. If $\beta=1$, then the expected utility of the cash flow takes the following form:

$$
E(U(X))= \begin{cases}E_{2}(\alpha)=\alpha(V+p-B)+B-p & \text { for } \alpha \geq \alpha_{V} \\ E_{1}(\alpha)=\alpha(\lambda(V+p)-B)+B-\lambda p & \text { for } \alpha<\alpha_{V}\end{cases}
$$

We see that it is a linear function of parameter $0 \leq \alpha \leq 1$. For $\alpha=0$, we obtain $E(U(X))$ $=B-\lambda p$ for $\alpha=\alpha_{V}$, we have $E(U(X))=B \frac{V S P}{V S P+p}$ and $E(U(X))=V S P$ for $\alpha=1$.

We will investigate five cases.

1. If $V S P+p>B$, then the functions $E_{1}$ and $E_{2}$ are increasing, and we obtain the optimum for $\alpha=1$ or $\alpha=\alpha_{V}$. But $B \frac{V S P}{V S P+p}<V S P$ in this case, and $\alpha=1$ is the optimal resale parameter.

2. If $V S P+p=B$, then for any $\alpha \in\left[\alpha_{V}, 1\right]$ we have $E(U(X))=V S P$. The function $E_{1}$ is increasing, so it obtains the maximum for $\alpha=\alpha_{V}$, and every $\alpha \in\left[\alpha_{V}, 1\right]$ is optimal.

3. If $V S P+p<B$ and $\lambda(V S P+p)>B$, then the function $E_{2}$ is decreasing and $E_{1}$ is increasing. So, the resale parameter $\alpha_{V}$ is optimal.

4. If $\lambda(V S P+p)=B$, then for any $\alpha \in\left[0, \alpha_{V}\right]$ we have $E(U(X))=B-\lambda p=\lambda V S P$. The function $E_{2}$ is decreasing, so it obtains the maximum for $\alpha=\alpha_{V}$ and every $\alpha \in\left[0, \alpha_{V}\right]$ is optimal. 
5. If $\lambda(V S P+p)<B$, then the functions $E_{1}$ and $E_{2}$ are decreasing, and we obtain the optimum for $\alpha=0$ or $\alpha=\alpha_{V}$. But $E_{2}\left(\alpha_{V}\right)=E_{1}\left(\alpha_{V}\right)<E_{1}(0)$ and $\alpha=0$ is optimal.

This completes the proof.

Example 3 (Prospect theory - example 1-2 continued). As based on Kahneman and Tversky's experiment in [12], the expected utility of the cash flow is equal to

$$
E(U(X))= \begin{cases}(\alpha V S P-1.4817(1-\alpha))^{0.88}+53.5312(1-\alpha)^{0.88} & \text { for } \alpha \geq \alpha_{V} \\ 53.5312(1-\alpha)^{0.88}-2.25(1.4817(1-\alpha)-\alpha V S P)^{0.88} & \text { for } \alpha<\alpha_{V}\end{cases}
$$

where $\alpha_{V}=\frac{1.4817}{V S P+1.4817}$. If the resale parameter $\alpha=0$, then the expected utility of the cash flow is equal to 50.3510 and when $V S P=20.5561$, we have $\alpha_{1}=0.0443$, $\alpha_{V}=0.0672$, and $\alpha_{2}=0.0673$. The expected utilities for such parameters are equal 50.2045 for $\alpha_{1}, 50.3509$ for $\alpha_{V}, 50.3510$ for $\alpha_{2}$, and 14.3018 for $\alpha=1$. So, we obtain two solutions for this optimisation problem: $\alpha=0$, and $\alpha=0.0673$.

Table 4. Optimal values of $\alpha$ and the maximum values of $E_{w+}(U(X))$ for different values of VSP

\begin{tabular}{|c|c|c|c|c|c|c|c|c|c|}
\hline \multirow{2}{*}{$V S P$} & \multicolumn{2}{|c|}{$x=20$} & \multicolumn{2}{|c|}{$x=55$} & \multirow{2}{*}{$V S P$} & \multicolumn{2}{|c|}{$x=20$} & \multicolumn{3}{c|}{$x=55$} \\
\cline { 2 - 6 } \cline { 7 - 9 } & $\alpha_{0}$ & $E_{w}(U(X))$ & $\alpha_{0}$ & $E_{w}(U(X))$ & & $\alpha_{0}$ & $E_{w}(U(X))$ & $\alpha_{0}$ & $E_{w}(U(X))$ \\
\hline 0 & 0 & 50.3495 & 0 & 42.3606 & 50 & 0.0422 & 52.2589 & 0.1081 & 47.0625 \\
\hline 10 & 0 & 50.3495 & 0 & 42.3606 & 60 & 0.0720 & 52.7100 & 0.1646 & 48.0544 \\
\hline 19.459 & 0 & 50.3495 & 0 or 0.1778 & 42.3606 & 70 & 0.1527 & 53.4729 & 0.2975 & 49.5275 \\
\hline 20.556 & 0 or 0.0673 & 50.3495 & 0.1699 & 42.7218 & 80 & 0.3021 & 54.8748 & 0.4909 & 51.8465 \\
\hline 30 & 0.0474 & 51.3086 & 0.1240 & 44.8442 & 90 & 0.4957 & 57.1721 & 0.6786 & 55.0942 \\
\hline 40 & 0.0385 & 51.8613 & 0.1020 & 46.1292 & 100 & 0.6753 & 60.3715 & 0.8131 & 59.0597 \\
\hline
\end{tabular}

Table 4 contains the solution to our optimisation problem for different values of $V S P$. The optimal values of the parameter $\alpha$ concerning the bounds of VSP are presented in Fig. 6. We examine two cases of a 60 -year-old man who bought the policy being 20 or 55 years old. We see that when $x=20$ for the acceptable values of VSP, we obtain regular results, similar to the ones for the classical utility case. But for smaller values of $V S P$, we can observe a different situation. For $0 \leq V S P \leq 20.556$, the optimal amount of resale parameter $\alpha_{0}=0$, i.e., the viatical contract has not been completed. The graph of the values of optimal $\alpha$ is not continuous at point $V S P=20.5561$ (Fig. 6). But when the insured bought the policy later, when he was 55 years old (compare Example 1), then $14.254 \leq V S P \leq 93.482$ and the discontinuity point $V S P=19.475$ lies in this interval. 


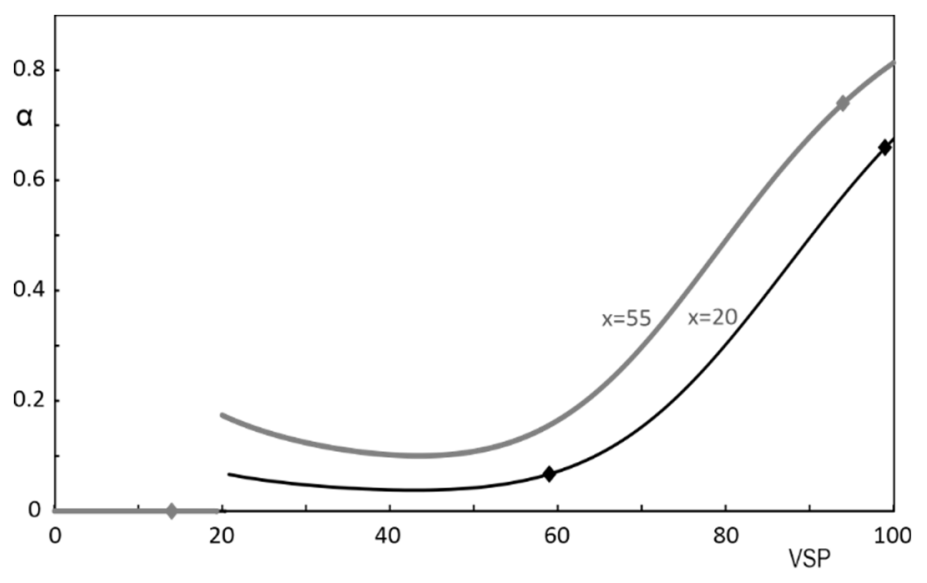

Fig. 6. The optimal values of the parameter $\alpha(C P T)$

Figure 7 contains the graphs of the values of the expected utility cash flow $E_{w+}(U(X))$ for the different values of the parameter $\alpha$ and fixed values of $V S P=80$. The maximal values of the expected utility cash flow $E_{w^{+}}(U(X))$ are indicated.

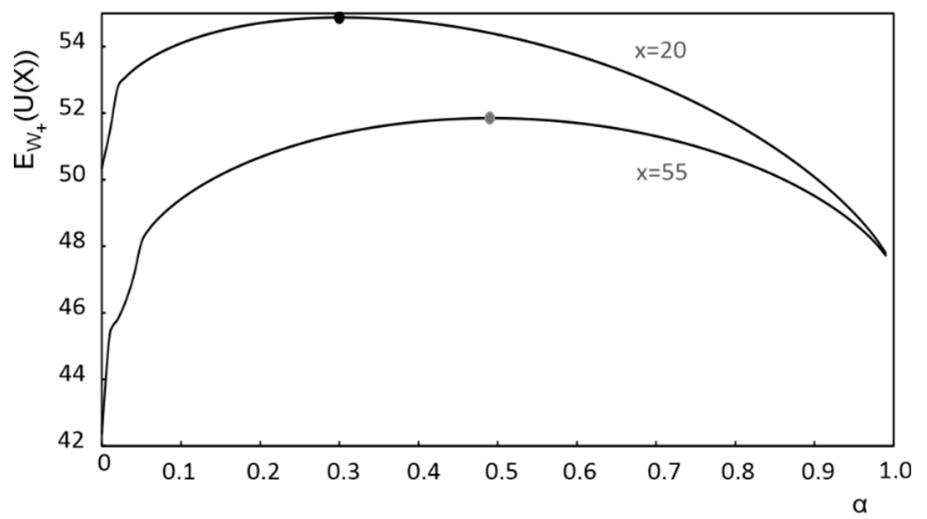

Fig. 7. The graph of $E_{w^{+}}(U(X))$ for different values of the parameter $\alpha$ and $V S P=80$

We can see that a longer duration of the policy results in a smaller optimal value of the parameter $\alpha$ and a higher value of the expected utility cash flow. The graph of the values of the expected utility cash flow $E_{w+}(U(X))$ for $V S P$ equals $15,19.4475$ (discontinuity point), and 25, respectively, and for small values of $\alpha$ when $x=55$ are presented in Fig. 8. The maximal values of the expected utility cash flow $E_{w+}(U(X))$ are indicated. These three values of $V S P$ are acceptable.

Let us assume that $\beta=1$ and $V S P=20.5561$, then the expected utility cash flow is equal to 


$$
E(U(X))= \begin{cases}-31.4934 \alpha+52.0495 & \text { for } 0.0672 \leq \alpha \\ -3.9462 \alpha+50.1974 & \text { for } \alpha<0.0672\end{cases}
$$

We see that $\lambda(V S P+p)<B$ and we have 5 th case. So, $\alpha=0$ is optimal resale parameter.

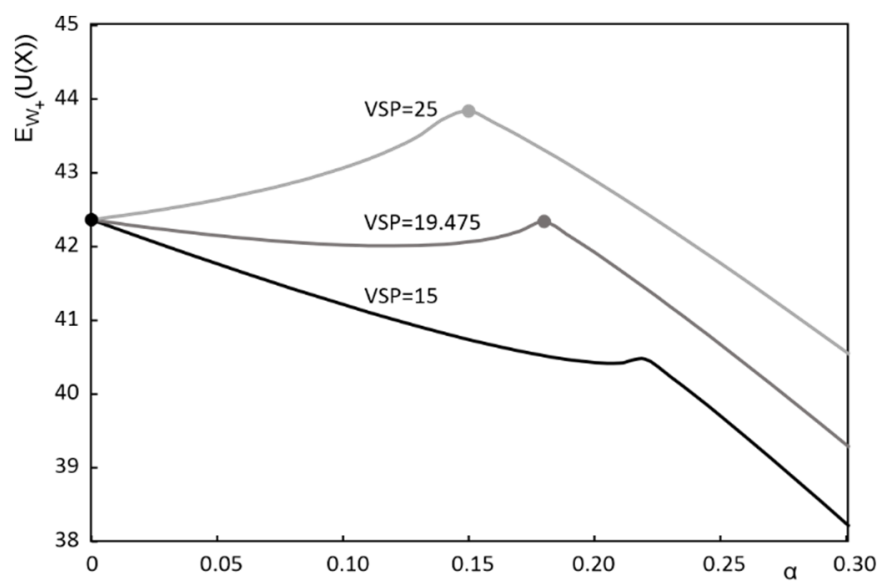

Fig. 8. Dependences of the expected utility cash flow on $\alpha$ for selected values of $V S P$ for $x=55$

\section{Conclusions}

The article defines the strategies of the insured in the case of resale of death rights. It shows that the optimum strategy of the insured depends on the amount the investor offers for the resignation from his or her death rights $(V S P)$. Note that the viatical settlement payment is directly influenced by the age at entry, the duration of the contract until the insured is sick with a serious illness and the type of dread disease. Thus, many components influence decision making, but under given circumstances, the discussed theories point to the optimal solution. In such a situation, the optimal choice of a resale parameter is affected by the way the insured person behaves under conditions of uncertainty. An optimal strategy is given for different kinds of human behaviour in risky conditions.

According to the expected value of cash flow, there is a $V S P_{0}$ for which each insured's decision is optimal (all $\alpha$ 's are just as "good"). If the viatical settlement payment proposed by the investor is lower, then the optimal decision of the insured is not to resell rights to the death benefit. If the investor offers a higher amount than $V S P_{0}$, the most profitable decision for the insured is to resell the whole insurance (the insured's expected profit is equal to viatical settlement payment $V S P$ ). In the case of utility theory 
and rank dependent expected utility theory, a higher value of VSP implies a greater value of the optimal resale parameter. When the insured proceeds according to prospect theory, for small $V S P$ values (up to some particular point) the insured practically make no profit while reselling insurance. This particular point is very interesting because it appears that for this particular VSP, we have two optimal solutions. Then, for increasingly higher value $V S P$ (up to this particular point), the value of the optimal resale parameter slowly falls to grow later on.

The theoretical results obtained in this paper are illustrated with numerical examples for a 60 -year old insured man, who becomes terminally ill with lung cancer. Because the range of VSP depends on his the age at entry, we considered two situations: an insured bought the whole-life insurance when he was 20-year old, and he bought insurance when he was 55-year old. The optimal values of resale parameters are summarised in Tables 5 and 6.

Table 5. The optimal values of $\alpha(x=20, t=40)$

\begin{tabular}{|l|c|c|c|c|c|c|c|c|}
\hline & & & & $\min$ & & & & $\max$ \\
\hline$V S P$ & 14.254 & 19.459 & 20.556 & 59.2 & 92.274 & 93.483 & 95.687 & 96.899 \\
\hline Expected value & 0 & 0 & 0 & 0 & 0 & 0 & {$[0,1]$} & 1 \\
\hline Utility theory & 0.07262 & 0.10519 & 0.11227 & 0.35499 & 0.51183 & 0.51660 & 0.52515 & 0.52976 \\
\hline RDEUT & 0.09124 & 0.12731 & 0.13502 & 0.38508 & 0.53825 & 0.54285 & 0.55108 & 0.55552 \\
\hline Prospect theory & 0 & 0 & 0 or 0.0673 & 0.06809 & 0.53979 & 0.56263 & 0.60291 & 0.62418 \\
\hline
\end{tabular}

Table 6 . The optimal values of $\alpha(x=55, t=5)$

\begin{tabular}{|l|c|c|c|c|c|c|c|c|}
\hline & $\min$ & & & & & $\max$ & & \\
\hline$V S P$ & 14.254 & 19.459 & 20.556 & 59.2 & 92.274 & 93.483 & 95.687 & 96.899 \\
\hline Expected value & 0 & 0 & 0 & 0 & {$[0,1]$} & 1 & 1 & 1 \\
\hline Utility theory & 0.10166 & 0.13737 & 0.14500 & 0.39339 & 0.54786 & 0.55253 & 0.56089 & 0.56541 \\
\hline RDEUT & 0.14250 & 0.18308 & 0.19153 & 0.44564 & 0.59163 & 0.59596 & 0.60370 & 0.60788 \\
\hline Prospect theory & 0 & 0 or 0.1778 & 0.16994 & 0.15746 & 0.71447 & 0.73227 & 0.76242 & 0.77775 \\
\hline
\end{tabular}

The amount of the optimal resale parameter is influenced by the period that elapses from the purchase of insurance to the moment of getting a serious illness. It can be seen (e.g., according to utility theory and the rules rank dependent expected utility theory) that the shorter the period, the higher the optimal value of the resale parameter for a particular VSP.

Also, for insurance which is short at the time of the insured person's illness, the range of acceptable values $V S P$ is greater. Then, according to the principles of prospect theory for small VSP values, the best decision of the insured is not to resell the rights to the death benefit. For the insurance which lasts for a long time when the insured person becomes ill, the acceptable bounds of VSP are narrower, but the values of the optimal 
resale parameter $\alpha$ are quite high. Then, the best decision is to resell parts of the insurance benefit, considering that the higher the value of $V S P$, the higher the optimal resale parameter.

We assume that three people of the same age have the same type of insurance, but each has a different approach to risk (i.e., by the UT, RDEUT, PT). Note that the largest difference in the size of the optimum resale parameter is observed for the insured who makes decisions according to the principles of prospect theory. Moreover, the optimal resale parameter for a person who proceeds according to rank dependent expected utility theory is higher than for an insured whose approach to risk is consistent with Utility Theory.

Research in optimisation strategies encourages further considerations. In particular, the impact of the rational choice factors that are the subject of behavioural finance research in the analysis of multi-person contracts.

\section{Acknowledgements}

The support of the grant scheme Non-standard multi-life insurance products with dependence between insured 2013/09/B/HS4/00490 is gladly acknowledged.

\section{References}

[1] Bhuyan V.B., Life Markets: Trading Mortality and Longevity Risk with Life Settlements and Linked Securities, Wiley, New York 2009.

[2] Bowers N.L., Gerber H.U., Hichmann J.C., Jones D.A., NesbitT C.J., Actuarial Mathematics, Illinois Society of Actuaries, Itasca 1986.

[3] Denneberg D., Lectures on Non-Additive Measure and Integral, Kluwer, Boston 1994.

[4] DęBICKa J., HeILPERn S., Investor's expected profit from viatical settlements, International Scientific Conference Applications of Mathematics in Economics, Szklarska Poręba, Poland, 30 August-3 September 2017, Conference Proceedings, Wydawnictwo Uniwersytetu Wrocławskiego, Wrocław 2017, 105-116, available at http://www.amse.ue.wroc.pl/papers/2017/ Debicka_Heilpern.pdf

[5] DęBicka J., Zmyślona B., Construction of multistate life tables for critical illness insurance. Influence of age and sex on the incidence of health inequalities, Silesian Statistical Review, 2016, 14 (20), $41-59$.

[6] DęBiCKa J., ZMYŚLONa B., Modelling of lung cancer survival data for critical illness insurances, Stat. Meth. Appl., 2019, 28 (4), 723-747.

[7] GATZERT N., The secondary market for life insurance in the United Kingdom, Germany, and the United States: comparison and overview, Risk Manage. Ins. Rev., 2010, 13 (2), 279-301.

[8] Haberman S., Pitacco E., Actuarial Models for Disability Insurance, Chapman and Hall/CRC, 1999.

[9] HEILPERN S., A rank-dependent generalization of zero utility principle, Ins. Math. Econ., 2004, 33, $67-73$.

[10] Hey J.D., Intermediate Microeconomics. People are different, McGraw-Hill, London 2004.

[11] Life Tables of Poland 2008, available at www.stat.gov.pl/en/topics/population/ life-expectancy/

[12] Kahneman D., TVersky A., Prospect theory. An analysis of decision under risk, Econometrica, 1997, 47, 313-327. 
[13] KaŁuszKa M., KRZESZOWIEC M., Pricing insurance contracts under cumulative prospect theory, Ins. Math. Econ., 2012, 50, 159-166.

[14] Nardon M., Pianca P., Behavioral premium principles, Dec. Econ. Fin., 2019, 42 (1), 229-257.

[15] NeERAJ S., Cashing Out Life Insurance. An Analysis of the Viatical Settlements Market, Santa Monica, California, RAND Corporation, RGSD-175, 2003. As of April 15, 2013: http://www.rand.org/pubs/rgs dissertations/RGSD175

[16] von Neuman J., Morgenstern O., Theory of Games and Economic Behavior, Princeton University Press, Princeton 1944.

[17] Quiggin J., A theory of anticipated utility, J. Econ. Beh. Org., 1982, 3, 323-343.

[18] SAMUelson P.A., A note on measurement of utility, Rev. Econ. Stud., 1937, 4 (2), 155-161.

[19] SLOAN F.A., NORTON E.C., Adverse selection, bequests, crowding out, and private demand for insurance: evidence from the long-term care insurance market, J. Risk Uncert., 1997, 15, 201-219.

[20] TVERSKy A., KAHNEMAN D., Advances in prospect theory: cumulative representation of uncertainty, J. Risk Uncert., 1992, 5, 297-323. 